\title{
Identification and Biochemical Characterization of Protein Phosphatase 5 from the Cantharidin-Producing Blister Beetle, Epicauta chinensis
}

\section{Xi'en Chen, Shumin Lü and Yalin Zhang *}

Key Laboratory of Plant Protection Resources and Pest Management of Ministry of Education, Northwest A\&F University, Yangling 712100, China; E-Mails: chenpp2006@ nwsuaf.edu.cn (X.C.); shuminlv@nwsuaf.edu.cn (S.L.)

* Author to whom correspondence should be addressed; E-Mail: yalinzh@ @ nwsuaf.edu.cn; Tel./Fax: +86-29-8709-2190.

Received: 28 November 2013; in revised form: 9 December 2013 / Accepted: 11 December 2013 / Published: 16 December 2013

\begin{abstract}
Protein phosphatase 5 (PP5) is a unique member of serine/threonine phosphatases which has been recognized in regulation of diverse cellular processes. A cDNA fragment encoding PP5 (EcPP5) was cloned and characterized from the cantharidin-producing blister beetle, E. chinensis. EcPP5 contains an open reading frame of $1500 \mathrm{bp}$ that encodes a protein of $56.89 \mathrm{kDa}$. The deduced amino acid sequence shares $88 \%$ and $68 \%$ identities to the PP5 of Tribolium castaneum and humans, respectively. Analysis of the primary sequence shows that EcPP5 has three TPR (tetratricopeptide repeat) motifs at its $N$-terminal region and contains a highly conserved $C$-terminal catalytic domain. RT-PCR reveals that EcPP5 is expressed in all developmental stages and in different tissues. The recombinant EcPP5 (rEcPP5) was produced in Escherichia coli and purified to homogeneity. The purified protein exhibited phosphatase activity towards pNPP (p-nitrophenyl phosphate) and phosphopeptides, and its activity can be enhanced by arachidonic acid. In vitro inhibition study revealed that protein phosphatase inhibitors, okadaic acid, cantharidin, norcantharidin and endothall, inhibited its activity. Further, protein phosphatase activity of total soluble protein extract from E. chinensis adults could be impeded by these inhibitors suggesting there might be some mechanism to protect this beetle from being damaged by its self-produced cantharidin.
\end{abstract}


Keywords: Epicauta chinensis; protein phosphatase 5; E. coli expression; in vitro inhibition

\section{Introduction}

Reversible phosphorylation of structural and functional proteins catalyzed by kinases and phosphatases is associated with the control of a wide variety of intracellular processes [1]. The numbers of catalytic subunits of protein phosphatases is nearly an order of magnitude lower than protein kinases [2]. Protein phosphatases are structurally and functionally diverse enzymes that comprise three major families: phosphoprotein phosphatases (PPPs), metal-dependent protein phosphatases (PPMs), and the aspartate-based phosphatases represented by FCP/SCP. The PPP family includes protein phosphatase 1 (PP1), PP2A, PP2B (or calcineurin), PP4, PP5, PP6 and PP7. These PPP family members are among the most highly conserved proteins known $[3,4]$.

In contrast to other PPP family members comprising isoforms encoded by different genes, PP5 is encoded by a single gene. Another unique structural characteristic of PP5 is that it possesses a highly conserved catalytic Ser/Thr phosphatase domain at the $C$-terminal half of protein as well as three to four tetratricopeptide repeat (TPR) domains at its $N$-terminal extension. These domains are believed to be involved in protein-protein interactions and activation of PP5 via specific binding to unsaturated fatty acids [5,6]. Unlike most of the PP1 and PP2A, purified PP5 exerts a low basal activity [7]. In mammals, PP5 regulates intracellular biological activities as diverse as cellular proliferation, differentiation, migration, survival and death, and DNA damage repair [8]. A recent study revealed that PP5 has an important role in the signaling mechanism associated with the diclazuril-induced merozoite apoptosis in Eimeria tenella [9]. To date, our knowledge of insect PP5 is still limited.

The blister beetles (Coleoptera: Meloidae) and some of the smaller oedemerid beetles (Coleoptera: Oedemeridae) are perhaps the most notorious of all poisonous insects due to their defensive toxin, cantharidin, which has been recognized as a potent PPPs inhibitor [10,11]. Mammal PP5 has been identified to be sensitive to several natural toxins, such as okadaic acid, microcystins, nodularin, calyculin A, tautomycin and cantharidin [12]. So far, there is no corresponding knowledge about whether insect PP5 is sensitive to these toxins. In addition, it is useful to investigate whether PP5 or other PPPs in cantharidin-producing beetles can be inhibited by cantharidin.

Herein, we undertook a study towards the identification and characterization of PP5 from a cantharidin-producing beetle, Epicauta chinensis. We analyzed the expression of this gene and the properties of its protein product along with its sensitivity to some PPPs inhibitors in vitro.

\section{Results and Discussion}

\subsection{Cloning and Sequence Analysis}

So far, PP5 genes from several insect species have been identified and published in the NCBI Database (National Center for Biotechnology Information, U.S. National Library of Medicine, Bethesda, MD, USA). However, no further study has analyzed their sequences or determined 
their function in biological processes. From our previously established transcriptome database of E. chinensis adults, we obtained the fragment containing the entire EcPP5 ORF of $1500 \mathrm{bp}$, which encodes a protein of 499 amino acids with the predicted molecular mass of $56897.92 \mathrm{Da}$ and theoretical isoelectric point of 5.91. The nucleotide sequence of EcPP5 cDNA was deposited in the GenBank (NCBI) database with the accession number KF765498.

Prosite analysis indicated the presence of three TPR (tetratricopeptide repeat) domains at its $N$-terminal portion which also exists in other known PP5s (Figure 1). Structural analysis of human PP5 revealed that access to the active site of the phosphatase domain is blocked by its TPR domains which maintains the low basal activity of PP5 [13]. The $C$-terminus consists of the highly conserved phosphatase domain containing three characteristic sequence motifs within the PPP family, GDxHG, GDxVDRG, and GNHE which play important roles in metal coordination, substrate binding and catalysis $[4,14,15]$. It also carries a nuclear targeting domain which is responsible for its transport into the nucleus. Additionally, a helix $\alpha \mathrm{J}$ motif was found at its $C$-terminus which could strengthen the association between the TPR domains and the phosphatase domain of PP5 [13] (Figure 1). BLAST analysis of EcPP5 deduced an amino acid sequence on NCBI that revealed a strong similarity with PP5s from other insect species with the highest identity being $88 \%$ to the PP5 of T. castaneum. It also shares a $68 \%$ identity to human PP5. The constructed phylogenetic tree shows the PP5 of E. chinensis, T. castaneum, and D. ponderosae from Coleoptera form a small cluster (Figure 2A).

Figure 1. Amino acid sequence comparison of insect Protein phosphatase 5 (PP5s). Alignment of the deduced amino acid sequence of EcPP5 was made with other insect PP5s from Tribolium castaneum (accession number XP 971407), Dendroctonus ponderosae (accession number AEE62915), Bombyx mori (accession number XP 004923376), and Culex quinquefasciatus (accession number XP 001850926). The positions of the three TPR (tetratricopeptide repeat) domains are indicated with lines of orange, green, and blue above the sequence. The catalytic domain and helix $\alpha \mathrm{J}$ motif are indicated with a red line and purple line above those sequences, respectively.

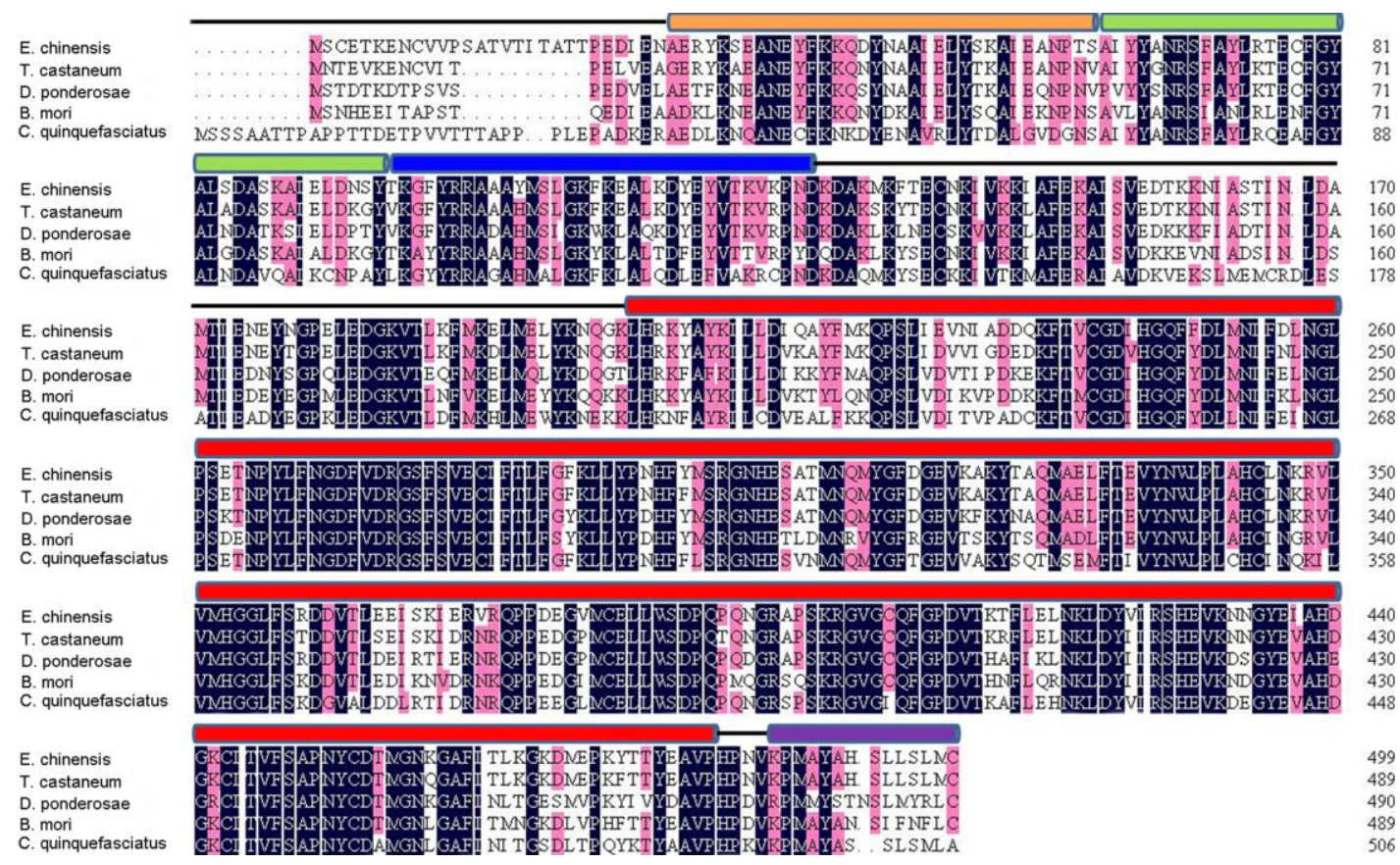


Figure 2. Phylogenetic tree construction and expression analysis of EcPP5. (A) Phylogenetic analysis of EcPP5 with other insect PP5s; (B) RT-PCR analysis of EcPP5 expression in different tissues of male and female adults; and (C) RT-PCR analysis of EcPP5 expression in various developmental stages. The actin gene (EcActin) was used as the reference gene.

A

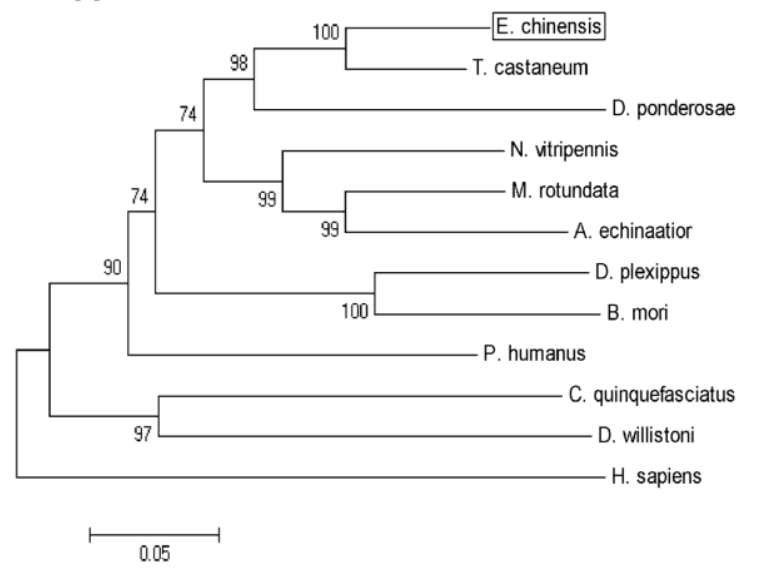

B

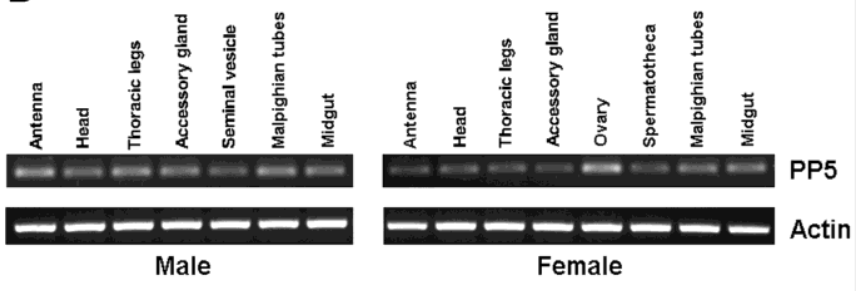

C

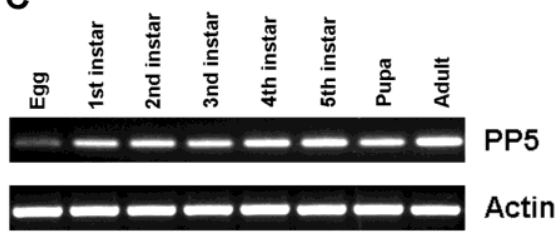

\subsection{Tissue- and Stage-Specific Expression Patterns of EcPP5}

Tissue-specific expression patterns of EcPP5 gene were analyzed in seven different tissues from male adults and eight different tissues from female adults, respectively, by using RT-PCR. Our results indicated that the EcPP5 gene was expressed in all tissues examined. In males, the expression levels among different tissues were nearly the same. While in the female, the EcPP5 transcript is more abundant in the ovary than in other tissues (Figure 2B). This may be the first report of tissue-specific expression of insect PP5.

Stage-specific expression patterns of the EcPP5 gene were determined for eggs, five different larval instars (1st, 2nd, 3rd, 4th and 5th), pupae and adults by using RT-PCR. We found that PP5 was expressed in all stages, with the lowest expression in eggs and the highest expression in adults (Figure 2C). In D. melanogaster, PP5 was expressed across the life cycle, but more highly expressed in the embryonic than at later developmental stages [16].

The transcript of EcPP5 was detectable in different tissues and developmental stages, suggesting that PP5 might participate in many biological processes in E. chinensis. However, more research will be needed to understand its role in biological activities in this beetle or other insects.

\subsection{Expression and Purification of Recombinant EcPP5}

Usually, expression of active PPP-type phosphatases in E. coli is difficult. E. coli-expressed PPP-type phosphatases are generally incorrectly folded and inactive. To facilitate their correct folding, chaperons, such as GroEL/GroES, have been used [17]. Since metal ions are indispensable for their phosphatase activity, a low concentration of metal ions (usually $2 \mathrm{mM} \mathrm{MnCl}_{2}$ ) needs to be externally added to the culture media [5]. PP5 genes from mammal, Plasmodium falciparum, and Trypanosoma brucei have been successfully expressed in E. coli $[7,18,19]$. However, there is no report for the heterologous expression of insect PP5. 
Herein, the EcPP5 ORF was subcloned into a modified expression vector pET43.1b and transformed into E. coli BL21(DE3)plysS cells for production of recombinant EcPP5 (rEcPP5). The EcPP5 gene was expressed as inclusion bodies in E. coli under $1.0 \mathrm{mM}$ IPTG (isopropyl $\beta$-D-thiogalactoside), at $37^{\circ} \mathrm{C}$ (data not shown), whereas, highly soluble rEcPP5 was produced under $0.1 \mathrm{mM}$ IPTG, at $18{ }^{\circ} \mathrm{C}$, with $2 \mathrm{mM} \mathrm{Mn}{ }^{2+}$ incorporated in the LB media. As expected, a band with an apparent molecular weight of $\sim 60 \mathrm{kDa}$ was detected on $12 \%$ SDS-PAGE (Figure 3) which is close to the predicted protein size from the deduced amino acid sequences. The soluble $N$-terminal His 6 tagged rEcPP5 was collected by a single step of affinity chromatography using Ni-NTA resin (Transgen, Beijing, China). High purity rEcPP5 protein (>90\%) was eluted by $50 \mathrm{mM}$ imidazole (Figure 3). After being dialyzed overnight at $4{ }^{\circ} \mathrm{C}$, the protein concentration was determined. Finally, $\sim 1.8 \mathrm{mg}$ of highly purified rEcPP5 was obtained from $500 \mathrm{~mL}$ cell culture.

Figure 3. Purification of rEcPP5. M: protein marker; TP: total soluble cell lysate; FT: flow-through elution; WD: wash-down elution; 1: $50 \mathrm{mM}$ imidazole elution; 2: $150 \mathrm{mM}$ imidazole elution.

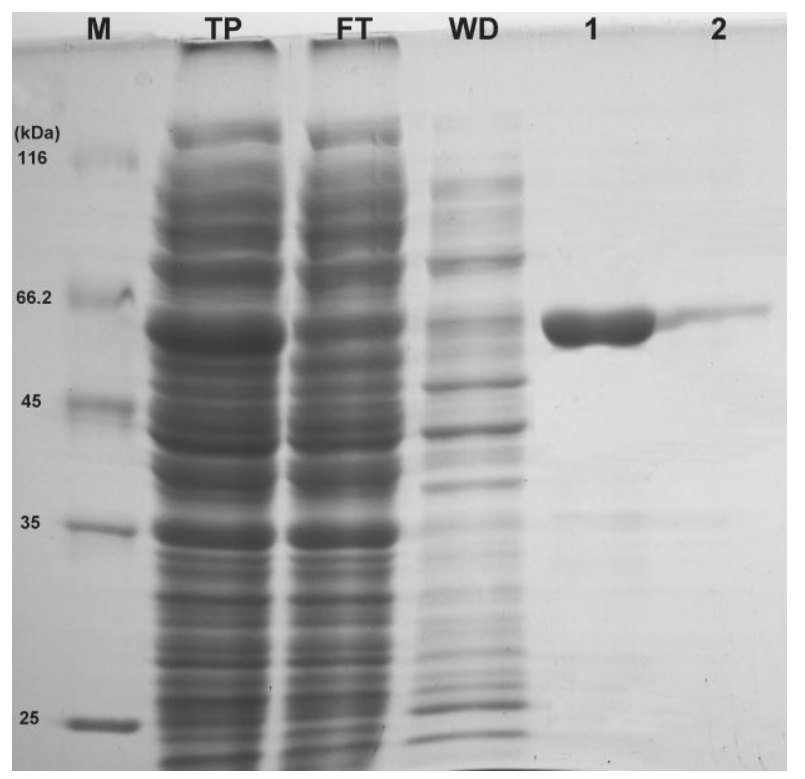

\subsection{Biochemical Characterization of the rEcPP5 Protein}

In contrast to other PPP family members, a unique property of PP5 is the stimulation of its phosphatase activity by unsaturated fatty acids. Using pNPP as the substrate, we found arachidonic acid stimulated the rEcPP5 activity up to 2.3-fold in a dose dependent manner with the maximum activation occurring at $200 \mu \mathrm{M}$ (Figure 4). This stimulation result was similar with those found on E. coli expressed Trypanosoma brucei PP5, 2.6-fold [18], and Plasmodium falciparum PP5, 2.0-fold [19]. It has been reported that the activation of mammalian PP5 activity by arachidonic acid varied from 4 to 25-fold [7,20]. We considered that the conformation of rEcPP5 expressed in E. coli might differ somewhat from the native one resulting in an increased intrinsic activity. We also carried out the arachidonic acid activation assay against total crude protein phosphatase extracted from E. chinensis adults. Total extracts showed phosphatase activity towards phosphopeptides, yet could not be stimulated by arachidonic acid (data not shown), which is probably due to the very low abundance 
of PP5 in the cells, or the activity is inhibited by intrinsic cantharidin. It is still notoriously difficult to purify the native PP5 to investigate whether arachidonic acid could stimulate its activity to higher folds.

Figure 4. Activation of rEcPP5 by arachidonic acid. Each point is the mean \pm SD of three independent assays.

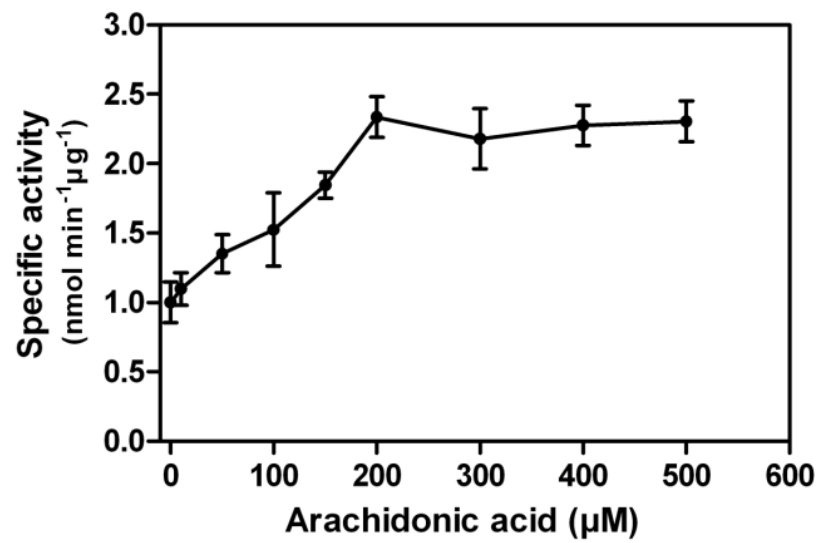

The optimal reaction $\mathrm{pH}$ or temperature was determined from a series of reactions in different $\mathrm{pH}$ assaying buffer or in $\mathrm{pH} 7.4$ assaying buffer at varying temperatures. The phosphatase activity of rEcPP5 first increased and then declined with ascending $\mathrm{pH}$ or temperature. The optimum reaction $\mathrm{pH}$ is about 7.4 (Figure 5A) and the optimum reaction temperature is around $55{ }^{\circ} \mathrm{C}$ (Figure $5 \mathrm{~B}$ ). The underlying mechanism for rEcPP5 possessing high phosphatase activity at relatively high temperatures is unknown. We presumed that rEcPP5 might undergo conformational changes with the increasing temperature which makes the substrate more easily accessible to its active site, while at a higher temperature its conformation begins to be destroyed leading to the loss of activity. So far, knowledge about the activity of PP5 in vivo at high temperatures is limited.

Figure 5. (A) Optimum reaction $\mathrm{pH}$; and (B) optimum reaction temperature determination of $\mathrm{rEcPP}$. Each point is the mean $\pm \mathrm{SD}$ of three independent assays.

A

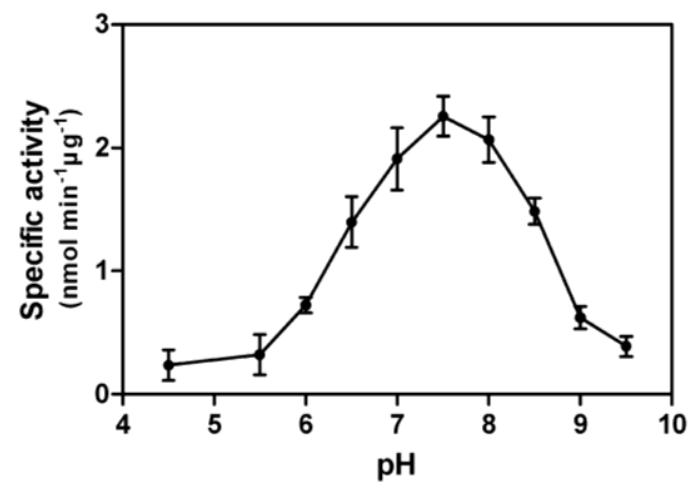

B

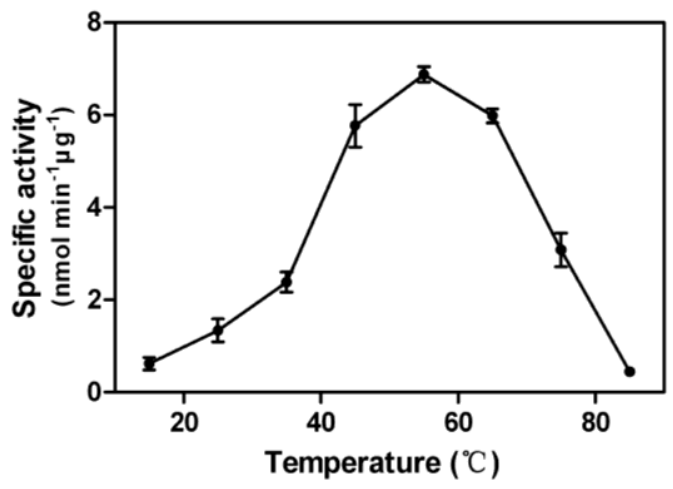

\subsection{Detection of rEcPP5 Phosphatase Activity in Vitro}

Enzyme-substrate reactions were carried out and kinetic properties were determined from Michaelis-Menten plots (Figure 6A,B), yielding $\mathrm{Km}$ of $13.32 \mathrm{mM}, \operatorname{Vmax}$ of $2.54 \mathrm{nmol} \mathrm{min}{ }^{-1} \cdot \mu^{-1}$ towards pNPP, and $\mathrm{Km}$ of $227.1 \mu \mathrm{M}$ and $\operatorname{Vmax}$ of $1.17 \mathrm{nmol} \mathrm{min}^{-1} \cdot \mu \mathrm{g}^{-1}$ against phosphopeptides. 
$k c a t$ and $k c a t / K m$ values were calculated as $2.54 \mathrm{~s}^{-1}$ and $0.19 \mathrm{mM}^{-1} \cdot \mathrm{s}^{-1}$ for $\mathrm{pNPP}$ and $1.17 \mathrm{~s}^{-1}$ and $5.15 \mathrm{mM}^{-1} \cdot \mathrm{s}^{-1}$ for phosphopeptides, respectively. Phosphopeptides have proved to be more specific substrates than pNPP for PPP-type phosphatases [21], and our results of lower $K m$ and higher $\mathrm{kcat} / \mathrm{Km}$ values also indicate that phosphopeptides are more specific and exert a stronger affinity to rEcPP5; however, we consider pNPP more qualified as a substrate in our assays. The reasons are as follows: (1) the method for using pNPP in the assays is simple to set up and easy to carry out; (2) the maximal catalytic activity of pNPP is usually much higher than that of phosphopeptides and can often approach that of a natural substrate [21]; and (3) the purified protein was taken as the enzyme source in our assays which circumvented the interaction between pNPP and other unexpected phosphatases.

Figure 6. Michaelis-Menten plots of rEcPP5 toward pNPP (A) and phosphopeptides (B). Each point is the mean \pm SD of three independent assays.

A

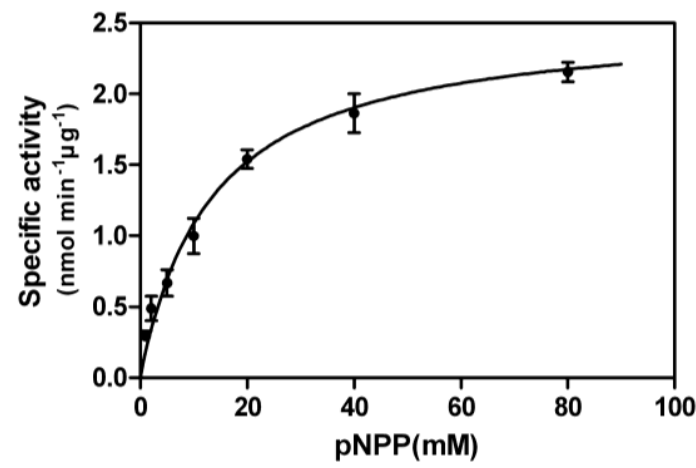

B

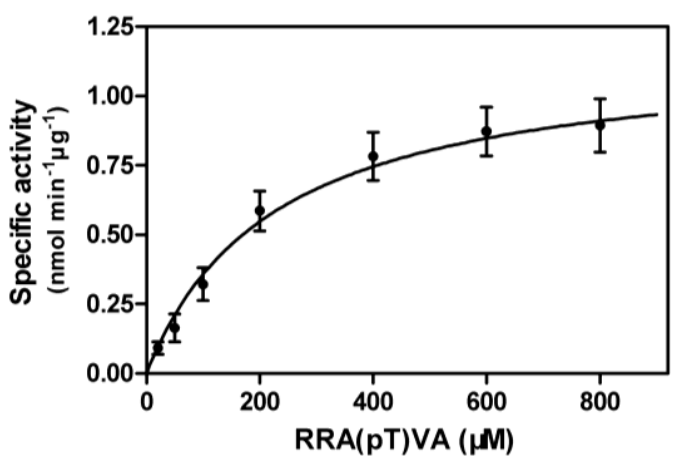

\subsection{Inhibition of Compounds on rEcPP5 and Crude PPP Extract}

Previous studies revealed that some naturally occurring toxins, such as okadaic acid, cantharidin, etc., could potently impair the phosphatase activity of both native and recombinant mammalian PP1, PP2A, PP4 and PP5 in vitro [12]. Here we found all four compounds exerted potent inhibitory effect on rEcPP5 in a dose-response response (Figure 7A). Okadaic acid inhibited rEcPP5 activity with an $I C_{50}$ value of $0.19 \mu \mathrm{M}$ that is significantly higher than the value reported for native bovine PP5 $\left(I C_{50}=3.5 \mathrm{nM}\right)$. However, the sensitivity of $\mathrm{rEcPP} 5$ to cantharidin, $I C_{50}=3.19 \mu \mathrm{M}$, was similar to recombinant mammal PP5 sensitivity to cantharidin $\left(I C_{50}=3.5 \mu \mathrm{M}\right)$ [12]. The $I C_{50}$ values of norcantharidin and endothall were 25.69 and $28.41 \mu \mathrm{M}$, respectively. There has been no report concerning the inhibitory effect of norcantharidin and endothall on PP5 before. Further study is needed to determine the sensitivity of native EcPP5 to these inhibitors. It will be of interest to compare the differences in the inhibitor-sensitivity between insect PP5 and mammal PP5.

It has been reported that cantharidin and endothall inhibited PPPs activity in the total soluble protein extract from Arabidopsis thaliana and Lemna paucicostata [22]. These four compounds showed an inhibitory effect on PPP activity in the extract of E. chinensis (Figure 7B). Okadaic acid was found to be the most potent inhibitor, with the residual phosphatase activity of only $8.79 \%$ at $10 \mu \mathrm{M}$. Cantharidin potently inhibited phosphatase activity in the extract, with the residual phosphatase activity of $19.36 \%$ at $10 \mu \mathrm{M}$. The inhibition of PPP activity by norcantharidin seemed as effective as inhibition by endothall, with no significant differences in the residual phosphatase activity 
at each concentration. This result is in agreement with the above results on the inhibitory effect of these compounds on rEcPP5.

We found cantharidin, together with the other three known PPP inhibitors, could block the activity of E. coli-expressed recombinant PP5 and PPP in the total protein extract from a cantharidin-producing beetle, E. chinensis. Given the highly conserved catalytic domain of the PPP family across the Eukaryota, it seems like that other members of PPP in E. chinensis are sensitive to cantharidin and other PPP inhibitors. Consequently, a question arises: how does this beetle prevent its own cantharidin accessing and affecting on its endogenous PPP? Work is currently in progress to answer this question.

Figure 7. Inhibition assay of compounds on rEcPP5 (A) and on PPP extract (B). Each point is the mean \pm SD of three independent assays.
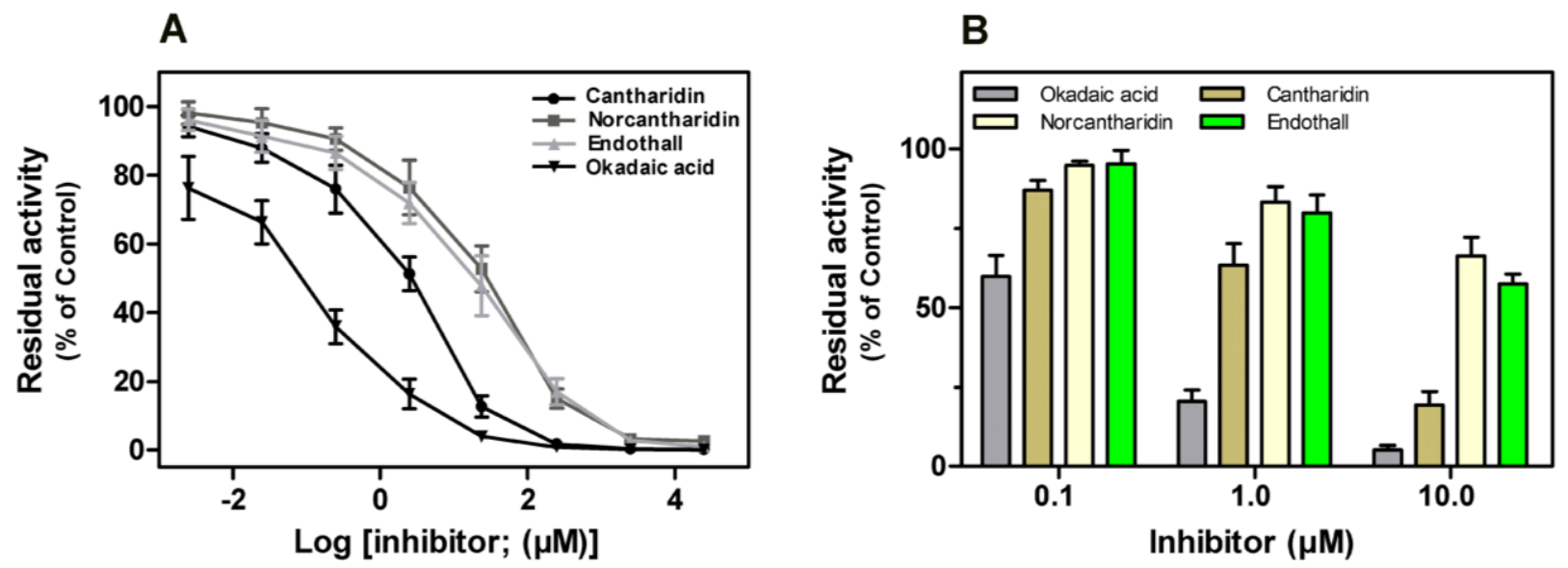

\section{Experimental Section}

\subsection{Insects and Chemicals}

Adults of the blister beetle E. chinensis were collected at their eclosion peak from soybean fields in Suide County, Shaanxi Province, China, on July 2011. All adults were kept in a chamber $(50 \mathrm{~cm} \times 50 \mathrm{~cm} \times 50 \mathrm{~cm})$ supplied with fresh lucerne (Medicago sativa) or soybean (Glycine max) leaves at $28.0 \pm 2.0{ }^{\circ} \mathrm{C}$, with a $14 \mathrm{~L}: 10 \mathrm{D}$ photoperiod. The larvae were reared individually in $150 \mathrm{~mL}$ plastic cups and fed with locust (Locusta migratoria) eggs under conditions of $30.0 \pm 1.0{ }^{\circ} \mathrm{C}$, $10.0 \pm 1.0 \%$ soil humidity, and a photoperiod of 16L:8D.

Okadaic acid, cantharidin, arachidonic acid, and pNPP were purchased from Sigma Chemical Corporation (St. Louis, MO, USA). Norcantharidin and endothall was purchased from Alfa Aesar Chemical Co. Ltd. (Haverhill, MA, USA). All other chemicals were of research grade or better and were obtained from commercial sources.

\subsection{Identification, Cloning, and Sequence Analysis of PP5 from E. chinensis}

The EcPP5 gene fragment was identified by searching the sequences in our previously established transcriptome database for E. chinensis adults via using the PP5 gene sequence of Tribolium castaneum (accession: XM_966314) as a probe. The putative EcPP5 fragment was further searched using BLASTX (NCBI) against the non-redundant database at NCBI to confirm its identity as a PP5 gene. 
The open reading frame (ORF) of EcPP5 was predicted via ORF Finder (NCBI). Primers for amplification of the ORF of EcPP5 were a sense primer 5'-CATATGCACCACCACCACCACCAC AGTTGTGAAACGAAAGAG-3' with Nde I restriction site (underlined) and 6 histidine codons (italic), and an antisense primer 5'-CTCGAGCTAGCACATTAGACTTAGTAAAG-3', containing the Xho I restriction site (underlined). Total RNA was extracted from adults using Trizol Plus (TaKaRa, Dalian, China) according to manual instructions. The quality and concentrations of RNA samples were examined by agarose gel electrophoresis and spectrophotometer analysis. RNA was digested with DNase I (TaKaRa, Dalian, China) and cDNA was synthesized using M-MLV RNaseH ${ }^{-}$ reverse transcriptase (Fermentas, Ontario, Canada) and stored at $-20{ }^{\circ} \mathrm{C}$ until use. PCR reaction was carried out on the S1000 TM Thermal Cycler (BioRad, Philadelphia, PA, USA) using ExTaq (TaKaRa, Dalian, China) under the following conditions: $95^{\circ} \mathrm{C}$ for $3 \mathrm{~min}, 30$ cycles of $95^{\circ} \mathrm{C}$ for $30 \mathrm{~s}, 60{ }^{\circ} \mathrm{C}$ for $30 \mathrm{~s}, 72{ }^{\circ} \mathrm{C}$ for $1.5 \mathrm{~min}$, followed by a final extension at $72{ }^{\circ} \mathrm{C}$ for $7 \mathrm{~min}$. The purified PCR products were inserted into pMD 19-T vector (TaKaRa, Dalian, China) and transformed into E. coli DH5 $\alpha$ (TaKaRa, Dalian, China), then sequenced (AuGCT, Inc., Beijing, China).

The amino acid sequence of EcPP5 was deduced from the obtained ORF cDNA sequence and the similarity analysis on the deduced amino acid sequence was performed using BLAST programs. The phylogenetic tree was constructed by MEGA 5.0 [23] by the maximum parsimony method using amino acid sequences of other insect PP5s, Tribolium castaneum (XP 971407), Dendroctonus ponderosae (AEE62915), Megachile rotundata (XP 003699533), Nasonia vitripennis (XP 001603324), Acromyrmex echinatior (EGI70292), Danaus plexippus (EHJ67807), Pediculus humanus (XP 002425763), Bombyx mori (XP 004923376), Culex quinquefasciatus (XP 001850926), and Drosophila willistoni (XP 002070260). The human PP5 (NP 006238) was employed as an outgroup. The ExPASy Compute $\mathrm{pI} / \mathrm{Mw}$ tool [24] was used to predict the molecular weight and isoelectric points of EcPP5. For the analysis of protein domains, the InterProScan 4 from EMBL-EBI [25] was used to search the InterPro collection of protein signature databases.

\subsection{Stage- and Tissue-Dependent Expression Analysis}

For stage-specific expression study, eggs, 1 st-5th instar larvae, pupae and adults were collected and stored at $-80{ }^{\circ} \mathrm{C}$ until use. For tissue-specific expression, antenna, head, thoracic legs, accessory glands, seminal vesicles, Malpighian tubules, and midgut were dissected from male adults on ice and stored at $-80{ }^{\circ} \mathrm{C}$ until use. Antenna, head, thoracic legs, accessory glands, ovary, spermatheca, Malpighian tubules, and midgut were dissected from females on ice and stored at $-80{ }^{\circ} \mathrm{C}$ until use. Total RNA was extracted as mentioned above. cDNA was synthesised from $1.0 \mu \mathrm{g}$ total RNA using a PrimeScript RT reagent kit with gDNA eraser (TaKaRa, Dalian, China). The forward primer, 5'-AAACGTGGTGTTG GTTGTCA-3', and the reverse primer, 5'-TTCCATCGTGAGCAATTTCA-3', were designed using Primer 3 [26]. The E. chinensis $\beta$-actin gene (accession: JQ764814) was used as a reference for RT-PCR analysis, with the forward primer, 5'-GCACCTGAAGAACATCCA-3', and the reverse primer, 5'-ACCAGAAGCATACAACGA-3'. Using 100-fold diluted cDNAs as templates, RT-PCR reactions were performed by a thermal cycle programme consisting under the following conditions: $95^{\circ} \mathrm{C}$ for $3 \mathrm{~min}, 28$ cycles of $95^{\circ} \mathrm{C}$ for $30 \mathrm{~s}, 60^{\circ} \mathrm{C}$ for $1 \mathrm{~min}$, and a final extension at $72{ }^{\circ} \mathrm{C}$ for $7 \mathrm{~min}$. The intensities of PCR products on $1 \%$ agarose gel were detected using a BioRad Gel Doc 2000 system 
(BioRad, Hercules, CA, USA). RT-PCR reaction was repeated at least 3 times, each with a new preparation of total RNA.

\subsection{Expression and Purification of Recombinant EcPP5}

The open reading frames (ORF) of EcPP5 with 6 histidine codons and Nde I site at $N$-terminal, and $X h o I$ site at $C$-terminal, were cloned into Nde I and Xho I (TaKaRa, Dalian, China) treated pET43.1b expression vector (Novagen, Madison, WI, USA), and transformed into E. coli BL21(DE3)plysS cells (Novagen, Madison, WI, USA). The positive transformed cells were amplified in LB medium containing $4 \mathrm{mM} \mathrm{Mn^{2+ }}, 100 \mu \mathrm{g} / \mathrm{mL}$ ampicillin and $34 \mu \mathrm{g} / \mathrm{mL}$ chloramphenicol. Recombinant EcPP5 (rEcPP5) expression was induced with $0.1 \mathrm{mM}$ isopropyl $\beta$-D-thiogalactoside (IPTG) when $A_{600}$ reached $0.4-0.6$ and this was continued for $24 \mathrm{~h}$ at $18^{\circ} \mathrm{C}$.

Cells were harvested by centrifugation at $7000 \times \mathrm{g}$ for $10 \mathrm{~min} 4{ }^{\circ} \mathrm{C}$ and sonication for $5 \mathrm{~min}$ on ice in Buffer A (20 mM Tris- $\mathrm{HCl}, \mathrm{pH}=8.0,20 \mathrm{mM}$ imidazole, $300 \mathrm{mM} \mathrm{NaCl}, 4 \mathrm{mM} \mathrm{MnCl}$ ) containing $0.1 \% \beta$-mercaptoethanol, $1.0 \mathrm{mg} / \mathrm{mL}$ isozyme and $1 \mathrm{mM}$ PMSF (phenylmethanesulfonyl fluoride). Debris was pelleted by centrifugation $\left(20,000 \times g\right.$ for $20 \mathrm{~min}$ at $\left.4{ }^{\circ} \mathrm{C}\right)$. The soluble fraction was loaded onto a Buffer A pre-equilibrated Ni-NTA affinity column (Transgen, Beijing, China) to bind the target protein; the non-target proteins were washed down with Buffer B. The target protein was eluted with 5 column volumes of Buffer A containing series concentrations of imidazole. Eluted solutions were subjected to $12 \%$ SDS-PAGE and stained with Coomassie Blue (Sigma-Aldrich, St. Louis, MO, USA). The rEcPP5 containing fractions were pooled and dialyzed against Buffer B (Buffer A + 50\% glycerol) overnight and then stored at $-20^{\circ} \mathrm{C}$. Protein concentration was assessed using the method of Bradford [27].

\subsection{Crude PPP Extraction}

The frozen adults were ground into a homogeneous state in ice-cold $20 \mathrm{mM}$ Tris $(\mathrm{pH}=7.4)$, $0.1 \% \beta$-mercaptoethanol, $1 \mathrm{mM}$ EDTA, $1 \mathrm{mM}$ benzamidine and $1 \mathrm{mM}$ (PMSF). This was then centrifuged at $12,000 \times \mathrm{g}$ for $30 \mathrm{~min}$ at $4{ }^{\circ} \mathrm{C}$. The supernatant extract was desalted using Sephadex G-25 spin columns (GE Healthcare, Boston, MA, USA) to remove the contaminating free phosphate as described in the instruction manual. Protein concentration was estimated using the Bradford method [27].

\subsection{Determination of Phosphatase Activity}

Activity of rEcPP5 against the inorganic phosphatase substrate pNPP was determined as described with slight modifications [28]. Assays were performed at $30{ }^{\circ} \mathrm{C}$ in a $100 \mu \mathrm{L}$ reaction containing $500 \mathrm{ng}$ purified rEcPP5, $200 \mu \mathrm{M}$ arachidonic acid and $20 \mathrm{mM}$ pNPP in $20 \mathrm{mM}$ Tris $(\mathrm{pH}=7.4), 1 \mathrm{mM}$ EDTA, $1 \mathrm{mM}$ EGTA, $0.1 \% \beta$-mercaptoethanol and $0.1 \%$ ethanol. After being pre-warmed to $30{ }^{\circ} \mathrm{C}$, the reaction was initiated by adding the pNPP. Assays were terminated after 15 min with $100 \mu \mathrm{L}$ of $5 \mathrm{~N} \mathrm{NaOH}$. Non-enzyme samples were taken as controls. Sample absorbance was measured at $A_{410}$ on Infinite $^{\circledR} 200$ PRO multimode micro-plate reader (Tecan, Austria). Kinetic parameters of rEcPP5 were estimated by using a Michaelis-Menten plot analysis of data obtained under the above assay conditions with pNPP concentrations of 1,2, 5, 10, 20, 40 and $80 \mathrm{mM}$. 
Phosphatase activity of rEcPP5 was also determined towards phosphopeptides using PPP assay kit (Promega, Madison, WI, USA) in half-area 96-well plates (Corning Inc., New York, NY, USA) following the manual's instructions. In brief, $500 \mathrm{ng}$ protein was mixed in an assay buffer of $50 \mathrm{mM}$ imidazole $(\mathrm{pH}=7.2), 200 \mu \mathrm{M}$ arachidonic acid, $0.2 \mathrm{mM}$ EGTA, $0.02 \% \beta$-mercaptoethanol, $0.1 \mathrm{mg} / \mathrm{mL}$ BSA, and pre-warmed to $30{ }^{\circ} \mathrm{C}$. Reaction was initiated after adding $100 \mu \mathrm{M}$ phosphopeptides, RRA(pT)VA to obtain a final volume of $50 \mu \mathrm{L}$ and incubated at $30{ }^{\circ} \mathrm{C}$ for $15 \mathrm{~min}$. The reaction was stopped by adding $50 \mu \mathrm{L}$ of molybdate dye/additive mixture and incubated for an additional 15 min at room temperature for color development. Control samples were determined without the enzyme. Sample absorbance was measured at $\mathrm{A}_{600}$ on an Infinite ${ }^{\circledR} 200$ PRO multimode micro-plate reader (Tecan, Austria). Kinetic parameters of enzymes were estimated by using a Michaelis-Menten plot analysis of data obtained under the above assay conditions with phosphopeptide concentrations of 20 , 50, 100, 200, 400, 600 and $800 \mu \mathrm{M}$. All assays were performed for three replications.

PPP activity in the extracts was measured using a PPP assay kit (Promega, Madison, WI, USA) as described above.

Using pNPP as substrate, the optimum reaction temperature of rEcPP5 protein was assayed for different temperatures and optimum reaction $\mathrm{pH}$ was determined in various $\mathrm{pH}$ substation solutions.

\subsection{Inhibition Assay}

Four candidate inhibitors were dissolved in dimethyl sulphoxide (DMSO) to provide stock solutions of $10 \mathrm{mM}$ for okadaic acid, $100 \mathrm{mM}$ for cantharidin, $200 \mathrm{mM}$ for norcantharidin and endothall, respectively. All solutions were diluted to the desired concentrations with assay buffer before use. Assays were carried out using pNPP as substrate. Before starting the reaction, the enzyme was incubated with the chemicals for $5 \mathrm{~min}$ at room temperature. A dose-response assay was used to determine the $I C_{50}$. The non-enzyme reaction was taken as the background control, while the non-inhibitor reaction was used as the full-activity control. An inhibition ratio was calculated as the percentage of $A_{410}$ values of the inhibition assay reaction divided by the full-activity control, having subtracted the background control $A_{410}$ value for both.

\section{Conclusions}

We isolated the PP5 gene from E. chinensis which shows a high sequence similarity with PP5 from other insects. The transcript of EcPP5 was detectable in different tissues of adults, as well as in all developmental stages, with the lowest level in eggs and highest level in adults. Soluble recombinant EcPP5 was obtained using an E. coli expression system with the addition of $\mathrm{Mn}^{2+}$ in the media. The purified rEcPP5 displayed phosphatase activity towards two substrates, pNPP and phosphopeptides. Moreover, similar to PP5 from other organisms, its activity could also be stimulated by arachidonic acid. Four known protein phosphatase inhibitors: okadaic acid, cantharidin, norcantharidin and endothall strongly inhibited rEcPP5 activity. We also found PPP activity in the total soluble protein of extract from this cantharidin-producing beetle could also be blocked by these four PPP inhibitors indicating there might be some protection mechanism to prevent this beetle from being damaged from its self-produced cantharidin. 


\section{Acknowledgments}

We thank Ming Jiang for help collecting and raising beetles. We sincerely appreciate J.R. Schrock (Emporia State University, Emporia, KS, USA) for revising the manuscript. This research was supported by the Special Fund for the Public Interest (Agriculture) by The Ministry of Science and Technology and The Ministry of Agriculture of China (200903052) and the "13115" Sci-Tech Innovation Project of Shaanxi Province (2007ZDKG-14).

\section{Conflicts of Interest}

The authors declare no conflict of interest.

\section{References}

1. Graves, J.D.; Krebs, E.G. Protein phosphorylation and signal transduction. Pharmacol. Ther. 1999, 82, 111-121.

2. Lander, E.S.; Linton, L.M.; Birren, B.; Nusbaum, C.; Zody, M.C.; Baldwin, J.; Devon, K.; Dewar, K.; Doyle, M.; FitzHugh, W.; et al. Initial sequencing and analysis of the human genome. Nature 2001, 409, 860-921.

3. Cohen, P.T.W. Overview of Protein Serine/Threonine Phosphatase. In Protein Phosphatases; Arino, J., Alexander, D.R., Eds.; Springer: Berlin, Germany 2004; pp. 1-20.

4. Shi, Y. Serine/threonine phosphatases: Mechanism through structure. Cell 2009, 139, 468-484.

5. Skinner, J.; Sinclair, C.; Romeo, C.; Armstrong, D.; Charbonneau, H.; Rossie, S. Purification of a fatty acid-stimulated protein-serine/threonine phosphatase from bovine brain and its identification as a homolog of protein phosphatase 5. J. Biol. Chem. 1997, 272, 22464-22471.

6. Das, A.K.; Cohen, P.T.W.; Barford, D. The structure of the tetratricopeptide repeats of protein phosphatase 5: Implications for TPR-mediated protein-protein interactions. EMBO J. 1998, 17, 1192-1199.

7. Chen, M.X.; McPartlin, A.E.; Brown, L.; Chen, Y.H.; Barker, H.M.; Cohen, P.T.W. A novel human protein serine/threonine phosphatase, which possesses four tetratricopeptide repeat motifs and localizes to the nucleus. EMBO J. 1994, 13, 4278-4290.

8. Hinds, T.D., Jr.; Sánchez, E.R. Protein phosphatase 5. Int. J. Biochem. Cell Biol. 2008, 40, 2358-2362.

9. Zhou, B.; Wang, H.; Zhao, Z.; Liu, M.; Yan, W.; Zhao, J.; Zhang, Z.; Xue, F. A novel serine/threonine protein phosphatase type 5 from second-generation merozoite of Eimeria tenella is associated with diclazuril-induced apoptosis. Parasitol. Res. 2013, 112, 1771-1780.

10. Carrel, J.E.; Doom, J.P.; McCormick, J.P. Identification of cantharidin in false blister beetles (Coleoptera, Oedemeridae) from Florida. J. Chem. Ecol. 1986, 12, 741-747.

11. Li, Y.M.; Casida, J.E. Cantharidin-binding protein: Identification as protein phosphatase 2A. Proc. Natl. Acad. Sci. USA 1992, 89, 11867-11870.

12. Honkanen, R.E.; Golden, T. Regulators of serine/threonine protein phosphatases at the dawn of a clinical era? Curr. Med. Chem. 2002, 9, 2055-2075. 
13. Yang, J.; Roe, S.M.; Cliff, M.J.; Williams, M.A.; Ladbury, J.E.; Cohen P.T.W.; Barford, D. Molecular basis for TPR domain-mediated regulation of protein phosphatase 5. EMBO J. 2005, $24,1-10$.

14. Zhuo, S.; Clemens, J.C.; Stone, R.L.; Dixon, J.E. Mutational analysis of a Ser/Thr phosphatase. Identification of residues important in phosphoesterase substrate binding and catalysis. J. Biol. Chem. 1994, 269, 26234-26238.

15. Ansai, T.; Dupuy, L.C.; Barik, S. Interactions between a minimal protein serine/threonine phosphatase and its phosphopeptide substrate sequence. J. Biol. Chem. 1996, 271, 24401-24407.

16. Brown, L.; Borthwick, E.B.; Cohen, P.T.W. Drosophila protein phosphatase 5 is encoded by a single gene that is most highly expressed during embryonic development. Biochim. Biophys. Acta 2000, 1492, 470-476.

17. Kelker, M.S.; Page, R.; Peti, W. Crystal structures of protein phosphatase-1 bound to nodularin-R and tautomycin: A novel scaffold for structure-based drug design of serine/threonine phosphatase inhibitors. J. Mol. Biol. 2009, 385, 11-21.

18. Chaudhuri, M. Cloning and characterization of a novel serine/threonine protein phosphatase type 5 from Trypanosoma brucei. Gene 2001, 266, 1-13.

19. Lindenthal, C.; Klinkert, M.Q. Identification and biochemical characterisation of a Protein Phosphatase 5 homologue from Plasmodium falciparum. Mol. Biochem. Parasitol. 2002, 120, 257-268.

20. Sinclair, C.; Borchers, C.; Parker, C.; Tomer, K; Charbonneau, H.; Rossie, S. The tetratricopeptide repeat domain and a $C$-terminal region control the activity of Ser/Thr protein phosphatase 5 . J. Biol. Chem. 1999, 274, 23666-23672.

21. McAvoy, T.; Nairn, A.C. Serine/threonine protein phosphatase assays. Curr. Protoc. Mol. Biol. 2010, 92, doi:10.1002/0471142727.mb1818s92.

22. Bajsa, J.; Pan, Z.; Dayan, F.E.; Owens, D.K.; Duke, S.O. Validation of serine/threonine protein phosphatase as the herbicide target site of endothall. Pestic. Biochem. Phys. 2012, 102, 38-44.

23. Tamura, K.; Peterson, D.; Peterson, N.; Stecher, G.; Nei, M.; Kumar, S. MEGA5: Molecular evolutionary genetics analysis using maximum likelihood, evolutionary distance, and maximum parsimony methods. Mol. Biol. Evol. 2011, 28, 2731-2739.

24. Compute pI/Mw. Available online: http://web.expasy.org/compute_pi/ (accessed on 26 November 2013).

25. InterProScan 4. Available online: http://www.ebi.ac.uk/Tools/pfa/iprscan/ (accessed on 26 November 2013).

26. Primer3. Available online: http://www.simgene.com/Primer3 (accessed on 26 November 2013).

27. Bradford, M.M. A rapid and sensitive method for the quantitation of microgram quantities of protein utilizing the principle of protein-dye binding. Anal. Biochem. 1976, 72, 248-254.

28. Takai, A.; Mieskes, G. Inhibitory effect of okadaic acid on the p-nitrophenyl phosphate phosphatase activity of protein phosphatases. Biochem. J. 1991, 275, 233-239.

(C) 2013 by the authors; licensee MDPI, Basel, Switzerland. This article is an open access article distributed under the terms and conditions of the Creative Commons Attribution license (http://creativecommons.org/licenses/by/3.0/). 